\title{
1. Employee commitment: an introduction and roadmap
}

John P. Meyer

Commitments are important. To wit, when politicians want to convince voters that they are serious about an issue, they make commitments. Similarly, businesses pledge their commitment to whatever quality they believe is important to customers in order to sell their products or services. And of course, couples make commitments to one another through the bonds of matrimony, often accompanied by elaborate celebrations by friends and family. As further testament to their importance, commitments can be frightening and sometimes politicians, businesses, and couples are reluctant to make them. Why do we seek, celebrate, and resist commitments? It is because commitments convey a sense of permanence. We want others to make commitments because it implies that they will persist in a course of action that will serve to benefit us in the future. We are reluctant to make commitments when we have reservations about whether we want to maintain a relationship or persist in a course of action in an uncertain future. Of course commitments can be broken and are sometimes made without a true intention to follow through. However, there is a stigma attached to breaking commitments, and lack of sincerity in making commitment breeds cynicism. This would not be the case if commitments were not important.

This book is about employee commitment, and the wealth of theory and research described in its pages attest to the fact that it too is important. The commitment of employees is only one aspect of workplace commitments more generally. Employers also make commitments to their employees, unions, customers, shareholders, regulatory bodies, and the like, and these commitments are also important. However, the vast majority of theory and research has focused on employee commitments, beginning initially with commitment to their organizations but extending to other entities (for example, occupation, union, team, supervisor, customer) or courses of action (for example, goals, decisions, policies, programs, change initiatives). Therefore, this book focuses on employee commitments and their implications for the targets of these commitments and the employees who make them.

Interest in employee commitment to organizations (that is, organizational commitment) began in earnest in North America, particularly the United States, in the 1960s and 1970s, although its roots can be traced back even further. This interest continues today, but the 1980s saw a branching of theory and research to include other targets of commitment. In the 1990s studies conducted in other parts of the world began to appear more regularly in the literature, and this trend burgeoned in the new millennium and shows no sign of waning. I will refrain from providing a more detailed historical review here because these are available elsewhere (e.g., Klein et al., 2009; Meyer et al., 2008; Mowday et al., 1982), including in Chapters 2, 3, and 4 in Part I of the current volume. Instead, I will focus on a few key issues that served as the impetus for this book and provide a roadmap to the content herein. 


\section{THE CONCEPTUALIZATION OF COMMITMENT}

Despite its importance in our everyday lives, few of us stop to ask what 'commitment' really means. I would hazard a guess that most organizational leaders, despite agreeing that they would like a committed workforce, have also rarely given serious thought to its definition. That is the job of academics, and many of us have been at it for a long time. Hence one might expect that there is a consensus on the definition and measurement of commitment. Not so! From the beginning, commitment has been examined from different disciplinary perspectives, using different methodologies and analytical procedures, and with different objectives. This continues to be the case today. Although some might argue that this failure to reach consensus reflects a lack of progress, I think the contents of this book belie that perspective. Indeed, we have learned a lot about commitment over the last 50-plus years that can and should be used to guide practice, despite academic disagreements. However, the disagreements themselves suggest that we still have much to learn, and should serve to fuel future investigation.

The authors of Part I of this Handbook, 'Conceptualization of Commitment', are thought leaders and have been actively involved in theory development and research for many years. Each has had a major impact on the field, both directly and indirectly through the large bodies of research they have stimulated. However, there are some fundamental differences in the approaches they have taken to the study of employee commitment. This is most evident in the divergence of opinion on the dimensionality of commitment, with Howard Klein and his student Hee Man Park arguing the case for unidimensionality in Chapter 2, and Natalie Allen stating the case for a multidimensional perspective in Chapter 3. Both chapters trace the historical roots of the differing perspectives, describe how it has guided research, and discuss its practical implications. Thomas Becker (Chapter 4) takes the less controversial perspective that employees can be committed to various work-relevant entities and/or courses of action. Although not contentious itself, the potential for multiple commitments raises questions about compatibility and conflict as well as the relative importance of these commitments as predictors of important outcomes. Addressing these questions requires a decision about how commitment should be conceptualized and measured, and the dimensionality issue again becomes front and center. The discussion of commitment in the remaining chapters of this book reflects how the issues of dimensionality and focus have been addressed by researchers as they investigate various substantive issues, including the development and consequences of commitment and the generalizability of theory and research findings across cultures. In the concluding chapter, I return to this issue, not with the objective of resolution, but to suggest how the energy generated by the debate might serve to guide future research.

\section{RELATED CONSTRUCTS}

Another challenge for both academics and practitioners is distinguishing commitment from related constructs (for example, motivation, engagement, embededdness, identification, and psychological contracts). What makes these constructs 'related', and why might they be considered a 'challenge' to those with an interest in commitment? I will have more to say on this issue in the concluding chapter, but the short answer for now is that they all 
relate to - and arguably influence - the same outcomes, including retention, attendance, job performance, citizenship behavior, counterproductive behavior, and/or well-being. The potential for construct redundancy is probably of greater concern for academics than it is for practitioners or their client organizations, but even the latter might find the terminology confusing, and this is arguably not conducive to the promotion of an evidence-based approach to management.

The authors of Part II, 'Related Constructs', have all been actively involved in theory development and research pertaining to these constructs and are well positioned to discuss the similarities to, differences from, and relations with commitment. Theory and research pertaining to work motivation has a longer history than commitment and it is legitimate to question whether there is really a difference between a committed and a motivated workforce. In Chapter 5, Gagné and Howard compare the two constructs from a self-determination theory (SDT) perspective and, although they distinguish the constructs primarily on the basis of focus (task versus organization), they also explain how key concepts within SDT (for example, need satisfaction) can be used to explain the motivating properties of commitments. Albrecht and Dineen discuss the similarities and differences between employee commitment and engagement in Chapter 6. Of the related constructs discussed in Part II, engagement is arguably the most controversial among commitment theorists. Purportedly introduced and popularized by human resources consulting firms (Macey and Schneider, 2008), some academics question whether it is simply 'old wine in new bottles', whereas others have embraced it and initiated elaborate programs of research designed, among other things, to demonstrate its unique contribution to understanding, predicting, and enhancing important organization- and employeerelevant outcomes.

In Chapter 7, Holtom describes theory and research pertaining to job embeddedness. Although introduced relatively recently in an effort to explain why employees stay with an organization, like commitment, job embeddedness also has implications for what employees do while on the job (for example, performance, citizenship behavior) as well as why they leave. The same is true of organizational identification, as discussed by Van Dick in Chapter 8, and psychological contracts, as discussed by Hansen and Griep in Chapter 9. Although there might be less concern in the latter cases with construct redundancy per se, there is some uncertainty about the nature and direction of their relations with commitment. The authors describe some interesting new developments in research pertaining to these constructs that have implications for their links to commitment. They also describe new and interesting research strategies that might be adapted in future commitment research (for example, use of manipulations to increase the salience of identities, or contract-relevant inducements that could have a bearing on the nature and/or focus of employees' commitments).

\section{MULTIPLE FOCI OF COMMITMENT}

Although commitment to the organization (that is, the employer) has been, and continues to be, the primary focus of research, it has long been recognized that employees can, and do, develop commitments to many different constituencies within the organization (for example, unit, supervisor, team) or outside it (occupation, union, clientele) (Becker, 1992; 
Morrow, 1983: Reichers, 1985; for more detail, see Chapter 4, this volume). These constituencies are typically individuals or groups with whom the employee interacts or belongs, but employees can also commit to a particular course of action (Becker, 1960; Kiesler, 1971; Salancik, 1977), or a proximal impetus to that action (for example, goal, decision, project, program, change initiative; see Neubert and $\mathrm{Wu}, 2009)$. Such commitments are of interest in their own right because of their implications for the relevant target (for example, participation in union activities; occupational retention and involvement; goal attainment; change effectiveness), but are often studied because of their implications for organizations. Acknowledging the multiple targets of commitment raises questions concerning the potential for conflict, compatibility, or synergy. Therefore, understanding the conditions that contribute to these different possibilities is of considerable importance for organizations and their employees. Organizations will want to minimize potential conflicts (for example, with unions or professions) and, where possible, strive for compatibility or synergy. In this era of instability and change, some organizations may find it difficult to establish long-term commitments with their employees and may seek substitutes such as commitment to supervisors, teams, or goals as a way of aligning employees' interests with their own (Meyer, 2009). Likewise, employees should benefit from alignment of goals and values among the targets of their commitment. Finally, as more research is conducted outside North America, and Western countries more generally, it is becoming apparent that cultural values and traditions can have an influence on the relative importance of commitment to targets within the organization (for example, supervisor, team) or outside it (for example, family, personal career). Therefore, understanding the foci of commitments, their interactions, and their influences is of importance to organizations operating internationally and/or with culturally diverse workforces.

The authors of Part III, 'Foci of Commitment', review theory and research pertaining to targets of commitment other than the organization. In Chapter 10, Meyer and Espinoza discuss commitment to one's occupation, or 'line of work', including both professional and non-professional occupations, and in Chapter 11, Vandenberghe examines social commitments to targets including supervisors, teams, and customers. In Chapter 12, Horsman, Gallagher, and Kelloway trace the history of theory and research pertaining to union commitment. The targets of commitment discussed in these chapters are all 'entities' with varying connections to the organization. Some (for example, supervisors and teams) are nested within the organization whereas others (for example, occupation, union, and customers) are external, but all are somewhat interrelated and create the potential for conflict, compatibility, or synergy with commitment to the organization. Finally, in Chapter 13, Meyer and Anderson review theory and research pertaining to action commitments; that is, commitment to a specific course of action, or to initiatives (for example, goals, policies, changes) that serve as the impetus to action. Although action commitments have generally received less attention than organizational, occupational, and social commitments, Meyer and Anderson argue that managing these commitments effectively might be particularly important under turbulent conditions that preclude the establishment of long-term relationships with an organization or social foci embedded within the organization. Although commitments to non-work foci (for example, family) are not specifically addressed in this section, they are discussed in several of the chapters in the section in Part VI on 'Commitment across Cultures'. 


\section{CONSEQUENCES OF COMMITMENT}

As noted above, commitments are important because they have implications for behavior. Interest in organizational commitment was arguably stimulated by its implications for retention (Mowday et al., 1982): employees who are committed to the organization should be less likely to leave voluntarily. Of course, keeping employees is not sufficient for organizations to be effective. Those employees must also attend regularly, perform their tasks effectively, contribute to a positive social milieu, and avoid engaging in behaviors that are counterproductive. Therefore, commitment theory and research gradually evolved to place more emphasis on on-the-job behavior and performance. Indeed, the impetus for the development of some of the multidimensional models discussed by Allen (Chapter 3 in this volume) was the notion that, regardless of how it is conceptualized, commitment to the organization should reduce turnover, but the implications for on-thejob behavior will vary depending on how it is experienced. Even more recently, researchers have begun to consider the implications of commitment, and its different forms and foci, for employees' own well-being (Meyer and Maltin, 2010).

Because the consequences of employee commitment are so central to interest in the construct itself, they are discussed to some extent in most of the chapters in this Handbook. However, the authors in Part IV, 'Consequences of Commitment', focus specifically on theory and research pertaining to the implications of commitments for the targets and the employees who make them. In Chapter 14, Gellatly and Hedberg focus on implications for employee withdrawal in the forms of turnover and absenteeism. As noted above, turnover has long been a focus of commitment researchers, but attendance is also important for organizations to achieve the full benefit of employment. In Chapter 15, Stanley and Meyer review the findings pertaining to two well-established forms of performance: task performance and organizational citizenship behavior. They also address the question of whether the nature of the commitment matters with regard to its implications for performance. In Chapter 16, Marcus looks at the flip-side of performance, namely counterproductive work behavior. Although of more recent interest, the implications of commitment, and the way it is experienced, on behaviors that are harmful to organizations and/or their employees is no less important. In the final two chapters in this section, the authors focus more on outcomes of relevance to employees themselves. In Chapter 17, Chris, Maltin, and Meyer consider implications for employees' physical and psychological well-being. In Chapter 18, Klein and Brinsfield address the role(s) that affect plays in the commitment process. They illustrate how employees' affective experiences can be impacted by their commitment, but acknowledge that they are probably implicated more broadly in the commitment process. The authors argue that affect has not received sufficient attention to date in commitment theory and research, and make important recommendations for how this situation might be rectified.

\section{DRIVERS OF COMMITMENT}

If commitment is important, then it is natural for scientists and practitioners to want to know how it develops. This is a tall order because there are arguably many factors that can contribute to the development of commitment. Despite the attention it has been 
given, early research conducted in the search for 'antecedents' was largely unsystematic and therefore uninformative. Indeed, Reichers (1985) referred to the variables included in early studies as a 'laundry list', and this was also apparent in early meta-analytic reviews (e.g., Mathieu and Zajac, 1990). A practitioner surveying this literature for clues as to how to foster stronger commitment would be hard-pressed to find anything meaningful. Fortunately, that situation has changed as theory and research have become more systematic and focused around basic principles that are arguably more generalizable and useful in guiding intervention strategies.

The chapters in Part V, 'Drivers of Commitment', are structured around broad themes or categories with inherent underlying principles. In Chapter 19, Bergman and Jean focus on the role(s) that individual differences can play in the development of commitment. It is perhaps not surprising that much of Bergman and Jean's discussion of individual differences focuses on how they interact with situational factors to influence commitment. Van Vianen, Hamstra, and Koen pick up on this theme in Chapter 20 and elaborate on the importance of person-environment $(\mathrm{P}-\mathrm{E})$ fit in its many forms (for example, person-job, person-organization, person-supervisor, person-team). The message in both of these chapters is that practitioners need to attend to both selection and work conditions, and to achieving a good match between the two. The remaining chapters in Part V focus more specifically on the work context. In Chapter 21, Jiang reviews theory and research pertaining to strategic human resource management (SHRM) practices, and more specifically on the implications of high-performance work systems (HPWSs) on commitment. Interestingly, much of the research in this tradition is conducted at an organizational level of analysis and makes a strong case for commitment as a mediator between HPWSs and various indices of organizational effectiveness. In Chapter 22, Trivisonno and Barling address the important role that leadership at various levels throughout the organization plays in shaping commitment. Among the many ways that leaders can foster commitment is by empowering employees, a practice addressed in more detail by Laschinger, Read, and Zhu in Chapter 23. In Chapter 24, Stinglhamber, Caesens, Clark, and Eisenberger discuss what has arguably been identified as one of the most important drivers of employee commitment, namely perceived organizational support (POS). Stinglhamber et al. note that organizational support is analogous to the commitment of the organization to its employees and that the strong relation between POS and employee commitment reflects the importance of social exchange and reciprocity. Importantly, they argue that supervisors can also contribute to the development of commitment, both directly through their own supportive behavior (perceived supervisor support), and indirectly by helping to foster POS. Finally, in Chapter 25, Bobocel and $\mathrm{Mu}$ explain the important role that justice perceptions have on the development and maintenance of employee commitment. Their treatment of justice provides an excellent example of how a set of principles firmly established in one domain (organizational justice) can help to inform another (organizational commitment). That is, having established a strong relation between fair treatment and commitment, practitioners can draw upon an extensive body of justice theory and research to guide the design and implementation of HRM systems. 


\section{COMMITMENT ACROSS CULTURES}

Most theory and the bulk of research on employee commitment originated in North America (the United States and Canada). Not surprisingly, the earliest expansion was to other Western countries. However, since the turn of the millennium, the picture has changed and published research is now coming regularly from other parts of the world. Admittedly, there is still far less research being conducted in non-Western than in Western countries, and there are large parts of the world for which there is still very little published research. Moreover, the research that does exist has largely been guided by Western theory; there is little in the way of new commitment theory being generated outside of the West (at least, theory that is easily accessible to those in the West who might be interested in new perspectives). Consequently, the authors in Part VI of this Handbook, 'Commitment across Cultures', were invited to write chapters with three objectives in mind. One was to provide a summary or representative sample of the commitment research conducted in a particular region of the world. Admittedly, this was a very challenging task because the regions selected were very large. A second objective was to identify ways that the culture(s) within these regions differ from the culture(s) in North America and might have a bearing on the nature, development or consequences of employee commitment. This task was also very challenging because, like North America, each region can be meaningfully divided into sub-regions with their own cultures, and even within these sub-regions there may be culture differences associated with language, ethnic origin, religion, economic conditions, climate, geography, and so on. The final objective was to identify gaps in existing theory and research that might be addressed in future research.

Wasti opens Part VI with Chapter 26 on the state of cross-cultural commitment research, and an astute analysis of the issues and challenges confronting researchers interested in examining commitment across countries and cultures. In Chapter 27, Felfe and Wombacher take on the challenging task of reviewing the commitment literature in Europe. As noted above, the challenge here is in synthesizing research coming from many different European countries with their multiple languages, histories, political systems, economies, and cultures. The task was perhaps facilitated to some degree by the fact that European researchers are well versed in the commitment literature and adept at conducting research. They also publish in journals that are easily accessible, many in English. In contrast, Newman and Wang (Chapter 28) and Dhir, Bremner and Datta (Chapter 29) not only took on the two most populous nations in the world - China and India, respectively - but also were confronted with situations where the amount of readily accessible published research is limited, there are multiple regions with different subcultures, and there are strong pressures for cultural change. In Chapter 30, Cohen faced similar obstacles in reviewing the literature in the Middle East; a region that is currently in turmoil and the focus of little published research. Most of the available research including Arab samples was conducted with Israeli Arabs who work under conditions that are quite unique in several respects. Finally, in Chapter 31, Arciniega provides a sampling of studies conducted in various countries within the large Latin American region. Although these countries differ in many ways, Arciniega points to some commonalities in cultural values, most notably high power distance and collectivism (Hofstede et al., 2010), and addresses the question of whether these differences from North American (that is, United 
States and Canadian) culture are reflected in the strength and nature of commitment as well as the effectiveness of HRM practices imported from North America.

\section{METHODOLOGICAL ISSUES IN COMMITMENT RESEARCH}

It is not uncommon for the complexities of the theories we develop, and the research questions we ask, to exceed the capacity of the methodological and analytic tools we have available to address them. This has certainly been the case with commitment theory and research. Fortunately, there are methodologists and statisticians who make it their business to develop new and better research tools. Importantly, there are others who bridge the two worlds and are able to apply the latest developments in methodology and analysis to address important substantive issues, and to share their expertise with others. The authors of Part VII of this Handbook are such individuals.

In Chapter 32, Vandenberg and Morelli provide a critique and update of Vandenberg and Lance's (2000) earlier recommendations for assessing measurement invariance across groups and/or over time. This is an important issue because many of the recommendations for future research offered throughout this Handbook involve the use of multi-wave longitudinal research intended to address causal relations proposed, but too seldom tested, within commitment theory. Other recommendations require the comparison of different groups (for example, cultural) with regard to the strength, development, or consequences of commitment. Providing meaningful answers to questions about changes over time or differences across groups requires some assurance that the instruments being used are measuring the same constructs in the same ways (that is, are invariant) over time and group.

Many of the most interesting questions we ask about employee commitment have to do with its development and consequences. Both imply causality, but very rarely do we, or are we able to, use the powerful experimental designs required to test causal hypotheses. More often than not we use non-experimental methods to detect covariation, one of the minimal requirements for causal inference. Occasionally we use longitudinal designs that allow for detection of covariation over time and partial control of extraneous variables. Fortunately, there have been a number of new developments in analytic procedures that can get us even closer to understanding how and why commitment develops and exerts its influence on behavior and well-being. In Chapter 33, Bentein describes how latent growth modeling (LGM) analyses can be applied to multi-wave longitudinal data to test a variety of the hypotheses implicit in our theories of commitment (for example, expectations prior to entry will influence the initial level of commitment that new employees experience, as well as the trajectory of change in their commitment over time; changes in commitment over time will be associated with changes in thoughts of quitting, and these changes, and perhaps the rate of change, will predict actual turnover).

In Chapter 34, van Olffen, Solinger, and Roe call for an even greater emphasis on time in the investigation of commitment. More specifically, they suggest how recent developments in temporal process research might be applied in the study of commitment. Further, they explain how taking a temporal process 'mindset' can have wide-ranging implications for commitment research, beginning with the conceptualization and measurement of the construct through to strategies for data collection and analysis. Although 
the approach is still very new and involves procedures that are likely to be somewhat controversial, it is a good illustration how new developments in methodology not only allow us to conduct better tests of existing theory, but can also challenge the status quo and stimulate us to think about the same phenomena from different perspectives.

Finally, early theories pertaining to multiple forms (for example, mindsets) and/or foci of commitment often included hypotheses concerning how these forms and foci might combine to influence behavior or other outcomes of interest. In the absence of analytic techniques well suited to testing these hypotheses, researchers often focus on the individual components (for example, mindsets or foci), or use analytic tools (for example, moderated multiple regression, median-split profile comparisons) that get closer to addressing the research question but have important limitations. In Chapter 35, Morin discusses new developments in person-centered research strategies that can be used to identify the different ways that commitment mindsets and/or foci combine to form profiles and interact to influence important outcomes. Like the temporal process approach discussed by van Olffen et al. in Chapter 34, taking a person-centered approach requires a different 'mindset' (Zyphur, 2009), but opens up a wide range of new research opportunities.

All of the analytic strategies described in Part VII are complex, but the authors provide clear descriptions of the underlying rationale and objectives, provide illustrative examples, and direct the reader to other sources for more details on the procedures required to use them. In short, they provide an excellent complement to the many useful recommendations for research offered throughout this book.

\section{A GUIDE FOR READERS}

Each of the chapters in this Handbook stands alone in providing a good overview of what we know about specific issues pertaining to employee commitment. However, the reader might find it helpful to consult the chapters in Part I on the conceptualization of commitment for the historical context surrounding these issues. Most authors also discuss how what we currently know can be used as a guide to practice, but also point to the gaps in our knowledge and identify important questions to be addressed in future research. The authors in Part VII describe some of the latest developments in methodology and analyses that can be used to address these questions. So I hope there will be something in the pages of this Handbook for all readers with an interest in the important topic of employee commitment, whether it is to get an up-to-date overview of theory and research, ideas for future research, or suggestions for building a more committed workforce. I will offer my attempt at a synthesis on all three counts in the concluding chapter.

\section{REFERENCES}

Becker, H.S. (1960). Notes on the concept of commitment. American Journal of Sociology, 66(1), 32-40.

Becker, T.E. (1992). Foci and bases of commitment: Are they distinctions worth making? Academy of Management Journal, 35(1), 232-244.

Hofstede, G., Hofstede, G.J. and Minkov, M. (2010). Cultures and Organizations: Software of the Mind (3rd edition). New York: McGraw-Hill. 


\section{Handbook of employee commitment}

Kiesler, C. (1971). The Psychology of Commitment. New York: Academic Press.

Klein, H.J., Molloy, J.C. and Cooper, J.T. (2009). Conceptual foundations: Construct definitions and theoretical representations of workplace commitments. In H.J. Klein, T.E. Becker and J.P. Meyer (eds), Commitment in Organizations: Accumulated Wisdom and New Directions (3-36). New York: Routledge/Taylor \& Francis.

Macey, W.H. and Schneider, B. (2008). The meaning of employee engagement. Industrial and Organizational Psychology: Perspectives on Science and Practice, 1, 3-30.

Mathieu, J.E. and Zajac, D.M. (1990). A review and meta-analysis of the antecedents, correlates, and consequences of organizational commitment. Psychological Bulletin, 108, 171-194.

Meyer, J.P. (2009). Commitment in a changing world of work. In H.J. Klein, T.E. Becker and J.P. Meyer (eds), Commitment in Organizations: Accumulated Wisdom and New Directions (37-68). Florence, KY: Routledge/ Taylor \& Francis Group.

Meyer, J.P. and Maltin, E.R. (2010). Employee commitment and well-being: A critical review, theoretical framework and research agenda. Journal of Vocational Behavior, 77(2), 323-337.

Meyer, J.P., Jackson, T.A. and Maltin, E.R. (2008). Commitment in the workplace: Past, present, and future. In C.L. Cooper and J. Barling (eds), Handbook of Organizational Behavior (Vol. 1, 35-53). Thousand Oaks, CA: Sage Publications.

Morrow, P.C. (1983). Concept redundancy in organizational research: The case of work commitment. Academy of Management Review, 8, 486-500.

Mowday, R.T., Porter, L.W. and Steers, R. (1982) Organizational Linkages: The Psychology of Commitment, Absenteeism, and Turnover. San Diego, CA: Academic Press.

Neubert, M.J. and Wu, C. (2009). Action commitments. In H.J. Klein, T.E. Becker and J.P. Meyer (eds), Commitment in Organizations: Accumulated Wisdom and New Directions (179-213). New York: Routledge/ Taylor \& Francis Group.

Reichers, A.E. (1985). A review and reconceptualization of organizational commitment, Academy of Management Review, 10, 465-476.

Salancik, G. (1977). Commitment and the control of organizational behavior and belief. In B. Staw and G. Salancik (eds), New Directions in Organizational Behavior (1-54). Chicago, IL: St Clair.

Vandenberg, R.J. and Lance, C.E. (2000). A review and synthesis of the measurement invariance literature: Suggestions, practices and recommendations for organizational research. Organizational Research Methods, 3, 4-70.

Zyphur, M. (2009). When mindsets collide: Switching analytical mindsets to advance organizational science. Academy of Management Review, 34, 677-688. 